\title{
Editorial: Are There Common Origins in Heterogeneous Dynamics and Structures in Ionic and Nonionic Systems?
}

\author{
Junko Habasaki ${ }^{1 *}$, Carlos Leon ${ }^{2}$ and K. L. Ngai ${ }^{3}$ \\ ${ }^{1}$ Materials Science, Schrödinger, K. K. 1-8-1, Tokyo, Japan, ${ }^{2}$ Dpto. Fisica de Materiales, Universidad Complutense de Madrid, \\ Madrid, Spain, ${ }^{3}$ CNR-IPCF, Pisa, Italy
}

Keywords: heterogeneity, glass transition, ionics, length scales, time scales

\section{Editorial on the Research Article}

Are There Common Origins in Heterogeneous Dynamics and Structures in Ionic and Nonionic Systems?

In the field of glass transitions, dynamic heterogeneity is often discussed in connection with the slowing down of dynamics for many kinds of nonionic systems. However, the existence of heterogeneous dynamics is not limited to the glass-forming nonionic materials near the glass transition. It was reported for ionic motions in ionically conducting glasses, as well as for ionic liquids (Ref. [1] and references therein). Interestingly, in these cases, it is also related to the cause of fast ionic motions. Furthermore, dynamical and related structural heterogeneity was commonly found in colloidal systems, metallic glasses, systems containing water, catalysts, biosystems, porous materials, and so on. That is, dynamic heterogeneity is widely observed both in ionic and nonionic systems. Although these systems tend to be discussed independently in each research field, they should have common underlying origins and mechanisms.

This Research Topic aimed to collect papers on heterogeneous dynamics and structures in the related fields. We sought to examine both the common origins and individual characteristics of ionic and nonionic systems. Furthermore, we expected to clarify the role of heterogeneity in the functioning of these systems.

The invitation to contribute to the Research Topic was answered by 23 authors. This Research Topic also benefited excellent reviewers.

As a result, related fields were widely covered, and many interesting views were gathered as summarized below.

Several papers are focused on the time and/or length scales of heterogeneity.

The paper by R. Das et al. has compared different time scales concerned with the heterogeneity of two glass-forming systems. The time at which the slope of the MSD vs. time in a log-log plot becomes unity is called $\tau \mathrm{D}$. The time at which the distribution of the displacements of the particles becomes Gaussian is called $\tau \mathrm{F}$. A similar time scale, $\tau \mathrm{H}$, is obtained via the van Hove correlation function. Their results imply that Fickian diffusion sets in at times much longer than the a-relaxation times in deeply supercooled liquids as suggested in earlier works [2].

It will be interesting to compare these results in glass-forming systems with those in ionic systems, where the changes between fast and slow dynamics are found in a longer time scale than in diffusion dynamics (Ref. [1] and references therein).

Saitoh and Kawasaki have examined dynamic heterogeneity in soft athermal particles (twodimensional disks) under shear by molecular dynamics simulations. They have clarified how the 
rheology, self-diffusion, and size of rigid clusters change with the externally imposed shear rate $\gamma$. and packing fraction of the disks $\phi$ in being related to its length scales. They argued the critical scaling of the diffusivity, as well as the size of rigid clusters near the jamming transition point. They also discussed the relation between diffusivity and size of rigid clusters with good quality of data.

The paper by Sasaki et al. discussed dielectric data for several glass-forming mixtures of a polymer with different polar liquids, including water. They investigated various relaxation processes and their temperature dependence when cooling down below the glass transition. The found relaxation processes were assigned to the segmental motion of the polymer, the motion of the polar molecules, and Johari-Goldstein (JG) $\beta$-relaxation. Based on the assignment of relaxation processes in the PVP-water mixture, they concluded that water molecules move cooperatively with PVP chains while PVP and alcohols move individually in the PVP-alcohols mixtures during a-relaxations.

The existence of different length scales is also discussed in the paper by Egami. The short-range atomic structure and the chemical nature of atomic bonds are not sufficient to explain liquid properties. The author illustrates the use of van Hove function (VHF), which is measurable experimentally, to examine the inherently dynamic nature of liquids. The dynamic two-body correlation via VHF reveals that there are coarse-grained medium-range density correlations common among various liquids and supersede chemical details of the system. By introducing these collective density fluctuations, the author tried to elucidate the origin of these medium-range density correlations and mentioned in passing that there is an interaction via longer-wavelength longitudinal waves which survive to high temperatures and modulate the local density (and not the detailed structure). It gives a hint to understand the universality of the interactions/waves in different liquids.

The temperature-dependent behavior of heterogeneous systems is also a key feature.

The paper by Xia et al. reports a study on the freezing of water and vitrification of $o$-terphenyl in various silica matrices.

\section{REFERENCES}

1. Habasaki J, Leon C, Ngai KL. Dynamics of glassy, crystalline and liquid ionic conductors - experiments, theories, simulations. New York, NY: Springer International (2017).

2. Szamel G, Flenner E. Time scale for the onset of Fickian diffusion in supercooled liquids. Phys. Rev. E (2006) 73:011504. doi:10.1103/PhysRevE. 73.011504
Performing calorimetric researches, they observe a single melting temperature $\mathrm{Tm}$ and single glass temperature $\mathrm{Tg}$ in confinements with monomodal pore size distributions, while there are $2 \mathrm{Tm}$ values and $2 \mathrm{Tg}$ values in confinements with bimodal pore size distributions. These experimental results are concerned with the coexistence of strong spatial heterogeneities over length scales of a few nanometers in the structure and dynamics, suggesting a close connection between them.

The role of heterogeneity in the functioning of materials is also addressed and will inspire the design of new functional materials.

The review article by Zanotto and Montazerian addresses the role of heterogeneous motion in crystallization processes. They have picked up lithium disilicate, water, and metallic glasses. In some works, the nucleation takes place in the high mobility, floppy region, contrary to the findings reported in other literature, where the nucleation in both water and metallic glasses occurred near the immobile domains. The relation between heterogeneity and nucleation exists in several systems; however, these results open new questions to the physics of nucleation.

At first sight, the common natures of heterogeneity among different systems may not be evident due to the difference in definitions of it. However, a careful comparison of ionic and nonionic systems reveals how remarkable common nature exists for heterogeneous dynamics and structures in these systems.

The relation between collective motions and single-particle motion in glass-forming materials is worth comparing with that between conductivity and self-diffusivity in ionics. The existence of dynamic heterogeneity can explain a variety of phenomena such as deviation from the Stokes-Einstein relation. We hope that this Research Topic can provide a useful resource for sharing knowledge and ideas with researchers in other related fields.

\section{AUTHOR CONTRIBUTIONS}

All authors have made a substantial, direct, and intellectual contribution to the work and approved it for publication.

Conflict of Interest: The authors declare that the research was conducted in the absence of any commercial or financial relationships that could be construed as a potential conflict of interest.

Copyright (c) 2021 Habasaki, Leon and Ngai. This is an open-access article distributed under the terms of the Creative Commons Attribution License (CC BY). The use, distribution or reproduction in other forums is permitted, provided the original author(s) and the copyright owner(s) are credited and that the original publication in this journal is cited, in accordance with accepted academic practice. No use, distribution or reproduction is permitted which does not comply with these terms. 